\title{
Evaluación del riesgo ergonómico por carga postural en estudiantes auxiliares de salud oral en una universidad del suroccidente colombiano
}

\author{
María del Pilar Zapata-Albán*, MSc. ${ }_{1}$ Katherine Volverás-Pimiento, Msc. ${ }_{2}$ \\ Facultad de Salud, Escuela de Rehabilitación Humana, Universidad del Valle, Santiago de Cali, Colombia \\ ,Terapias Integradas, Gestión 24-7 Seguros Bolivar, Santiago de Cali, Colombia
}

Recibido: 2 de noviembre del 2016 Aprobado: 6 de diciembre del 2016

*Autor de correspondencia: María del Pilar Zapata Albán. Calle 20 n. 154-34 casa 12 Peyares I. Cali, Valle del Cauca, Colombia. Fax: 5185658, Teléfono: 5185658-59, Cel: 3148968729. Correo electrónico: maria.zapata@correounivalle.edu.co

Cómo citar este artículo: Zapata-Albán M del P, Volverás-Pimiento K. Evaluación del riesgo ergonómico por carga postural en estudiantes auxiliares de salud oral en una universidad del suroccidente colombiano. Rev Nac Odontol. 2017;13(25):43-55. doi: http://dx.doi.org/10.16925/od.v13i25.1881

Resumen. Introducción: las lesiones musculoesqueléticas tienen un enorme y creciente impacto en el mundo al ser la mayor causa de dolor y discapacidad por sobrecarga muscular en el trabajo. Objetivo: determinar el riesgo ergonómico producido por la carga postural a la que está expuesta la población objetivo. Método: estudio observacional descriptivo de corte transversal. Se abordó una muestra por conveniencia de quince personas, estudiantes del programa Auxiliar de Salud Oral de una universidad del suroccidente colombiano; se aplicó encuesta sociodemográfica, análisis de puesto de trabajo y el método Rapid Upper Limb Assessment (RULA), cuya metodología divide el cuerpo en dos grupos: el grupo A, que incluye los miembros superiores (brazos, antebrazos y muñecas), y el grupo B, que comprende piernas, tronco y cuello; para ello, se realizaron dos registros fílmicos simultáneos cuando cada participante realizaba el procedimiento de profilaxis dental. Resultados: la información recolectada permitió identificar los pasos de mayor demanda física y los trastornos del sistema musculoesquelético producidos por la carga postural a la que está expuesta la población en la realización del procedimiento, y permitió identificar de manera específica los segmentos corporales comprometidos y el grado de compromiso. Conclusiones: existe riesgo ergonómico en los estudiantes auxiliares de salud oral, pues se encuentran en un nivel 4 de actuación, lo que significa que la tarea "realizar el procedimiento de profilaxis" requiere cambios urgentes, debido a la exposición a factores de riesgo biomecánicos que pueden ocasionar trastornos en la salud, especialmente en el sistema musculoesquelético.

Palabras clave: ingeniería humana, medicina del trabajo, riesgos laborales, profilaxis dental, sobrecarga postural. 


\title{
Ergonomic risk assessment for postural load in oral health assistance students at a university in southwestern Colombia
}

\begin{abstract}
Introduction: Musculoskeletal injuries have an enormous, growing impact on the world for being the main cause of pain and disability due to muscle overload at work. Aim: To determine the ergonomic risk produced by the postural load to which the target population is exposed. Method: Cross-sectional descriptive observational study. A convenience sample of 15 people, students of the Oral Health Assistance Program at a university in southwestern Colombia, was drawn; we conducted a sociodemographic survey, a workstation analysis, and the Rapid Upper Limb Assessment (RULA) method, whose methodology divides the body into two groups: Group A, including the upper limbs (arms, forearms and wrists), and group B, comprising legs, trunk and neck. For this, two simultaneous film records were kept when each participant performed the dental prophylaxis procedure. Results: The information collected allowed to identify the most physically demanding steps and the musculoskeletal system disorders produced by the postural load to which the population is exposed in performing the procedure, and allowed to identify the specific body segments compromised and the degree of compromise. Conclusions: There is an ergonomic risk in oral health assistance students, since they are at an action level of 4. This means that the task of "performing the prophylaxis procedure" requires urgent changes due to the exposure to biomechanical risk factors that may cause health disorders, especially in the musculoskeletal system.
\end{abstract}

Keywords: human engineering, occupational medicine, occupational hazards, dental prophylaxis, postural overload.

\section{Avaliação do risco ergonômico por carga postural em estudantes auxiliares de saúde oral numa universidade do sudoeste colombiano}

Resumo. Introdução: as lesões musculoesqueléticas têm grande e crescente impacto no mundo ao ser a maior causa de dor e deficiência por sobrecarga muscular no trabalho. Objetivo: determinar o risco ergonômico produzido pela carga postural à qual a população-alvo está exposta. Método: estudo observacional descritivo de corte transversal. Abordou-se uma amostra por conveniência de 15 pessoas, estudantes do programa Auxiliar de Saúde Oral de uma universidade do sudoeste colombiano; aplicou-se um questionário sociodemográfico, análise de posto de trabalho e do método Rapid Upper Limb Assessment (Rula), cuja metodologia divide o corpus em dois grupos: o grupo A, que inclui os membros superiores (braços, antebraços e pulsos), e o grupo B, que compreende pernas, tronco e pescoço; para isso, realizaram-se dois registros fílmicos simultâneos quando cada participante realizava o procedimento de profilaxia dental. Resultados: a informação coletada permitiu identificar os passos de maior demanda física e os transtornos do sistema musculoesquelético produzidos pela carga postural à qual a população está exposta na realização do procedimento, e permitiu identificar de maneira específica os segmentos corporais comprometidos e o grau de compromisso. Conclusões: existe risco ergonômico nos estudantes auxiliares de saúde oral, pois se encontram num nível 4 de atuação, o que significa que a tarefa "realizar o procedimento de profilaxia" requer mudanças urgentes, devido à exposição a fatores de riscos biomecânicos que podem ocasionar transtornos na saúde, especialmente no sistema musculoesquelético.

Palavras-chave: engenharia humana, medicina do trabalho, riscos profissionais, profilaxia dental, sobrecarga postural. 


\section{Introducción}

Las lesiones musculoesqueléticas tienen un enorme y creciente impacto a escala mundial, desde la perspectiva de la productividad y la economía de la industria. Son la principal causa de dolor y discapacidad, y debido a su alta prevalencia y a su asociación con otras morbilidades, tienen un importante impacto socioeconómico [1]. Según datos reportados por Kumar [2], cerca del 58\% de la población mundial mayor de 10 años de edad pasa un tercio de su tiempo en el trabajo, generando 21,6 trillones de dólares como producto interno bruto que sostiene la economía mundial; así mismo, se estima que del 30 al 50\% de los trabajadores están expuestos a riesgos ocupacionales que les pueden generar lesiones musculoesqueléticas [3].

En Colombia, la patología musculoesquelética representa el $65 \%$ del total de diagnósticos de enfermedades profesionales en el régimen contributivo, con lo que constituye la primera causa de morbilidad profesional, como se evidencia en los datos presentados por el Ministerio de Salud y Protección Social en el 2002 [4].

La sobrecarga postural en el trabajador se caracteriza porque este permanece fuera de la posición corporal neutra por un tiempo determinado, lo que favorece la presencia de sintomatología de dolor, inflamación, disestesias, parestesias y limitación para realizar su trabajo. Incluso, la sobrecarga postural llega a impedir la realización de actividades cotidianas y obliga al trabajador a solicitar incapacidad temporal para el trabajo, lo que genera ausentismo, disminución en la productividad, pérdidas económicas y, principalmente, daños importantes a la salud [5].

Son varias las investigaciones en ergonomía que se han realizado con el personal de la salud [6-8], específicamente en odontología $[9,10]$, pero fue solo en el 2006 cuando se encontró una correlación entre posturas de trabajo y dolor postural durante las prácticas clínicas de estudiantes en una Facultad de Estomatología [11]. Sin embargo, estos hallazgos se dieron en odontólogos o practicantes de odontología, pero no en los auxiliares de salud oral, quienes en esa época trabajaban con el odontólogo, acompañándolo y asistiéndolo durante los diferentes procedimientos, incluyendo el de profilaxis dental. Actualmente, se observa que el auxiliar se ha desligado del trabajo a cuatro manos, que ahora es realizado al mismo tiempo por un operador y un ayudante en el mismo campo operatorio, lo cual hace que estén expuestos a peligros biomecánicos que, sumados a otros, pueden generar una combinación de probabilidad y severidad que redunde finalmente en una afectación osteomuscular.

\section{Diseño}

Este estudio es descriptivo, observacional de corte transversal de una muestra poblacional en un solo momento temporal, es decir que permite identificar los factores de riesgo ergonómicos a los que pueden estar expuestos los estudiantes del programa Auxiliar de Salud Oral en un momento dado, con cifras exactas respecto a posiciones del cuerpo y características particulares del medio, la actividad y el sujeto mismo.

\section{Población}

Se utilizó un muestreo por conveniencia conformado por un total de quince estudiantes, todos matriculados en la asignatura Clínica de Prevención II, de las jornadas diurna y nocturna del programa Auxiliar de Salud Oral de la Escuela de Odontología de la Universidad del Valle, durante el primer semestre del 2013.

\section{Criterios de inclusión}

- Estudiantes de las jornadas diurna y nocturna del programa Auxiliar de Salud Oral de la Escuela de Odontología de la Universidad del Valle que se encuentren matriculados en la asignatura Clínica de Prevención II.

- Estudiantes mayores de edad.

- Estudiantes que usan su mano derecha como dominante, debido a que tienen una destreza en ese miembro superior sobre el otro y a que el espacio y los equipos están diseñados y distribuidos para personas con esa lateralidad.

- Estudiantes que acepten participar de manera voluntaria en el estudio y firmen el consentimiento informado, al igual que sus respectivos pacientes.

\section{Criterios de exclusión}

- Retiro voluntario del estudio. 
- Alguna lesión física que afecte la adecuada ejecución de un procedimiento de profilaxis dental durante el tiempo en que se lleve a cabo el estudio.

\section{Instrumentos de evaluación}

Se hizo una encuesta sociodemográfica en la que se obtuvo información sobre: datos de identificación, existencia o no de sintomatología, percepción de la ejecución del procedimiento de profilaxis dental, apreciación sobre la unidad odontológica y sus componentes, y condiciones ambientales del puesto de trabajo, con el fin de determinar el perfil ocupacional y contribuir en el análisis de la actividad y de los factores medioambientales a los que está expuesto el estudiante.

Se realizó un análisis y descripción del puesto de trabajo [12] del auxiliar de salud oral, con el fin de obtener información que permita identificar de manera completa y precisa qué hace el trabajador, cómo lo hace y para qué lo hace, al igual que las destrezas o exigencias que emplea para hacerlo; así, se reconoce la complejidad de las tareas, las operaciones y los pasos dentro del puesto, y se identifica el grado de complejidad de cada conjunto de acciones básicas y actividades que el trabajo exige a la persona que lo desempeña, información esencial para la aplicación del método RULA.

Se aplicó el método Rapid Upper Limb Assessment (RULA), desarrollado por los doctores McAtamney y Corlett, de la Universidad de Nottingham en 1993 (Institute for Occupational Ergonomics), para evaluar la exposición de los trabajadores a factores de riesgo que pueden ocasionar trastornos en los miembros superiores del cuerpo: posturas, repetitividad de movimientos, fuerzas aplicadas y actividad estática del sistema musculoesquelético [13].

Este método divide el cuerpo en dos grupos: el grupo A incluye los miembros superiores (brazos, antebrazos y muñecas) y el grupo B comprende piernas, tronco y cuello. Mediante las tablas asociadas al método, se asigna una puntuación a cada zona corporal (piernas, muñecas, brazos, tronco) para, en función de dichas puntuaciones, asignar valores globales a cada uno de los grupos A y B.

La clave para asignar las puntuaciones es la medición de los ángulos que forma cada parte del cuerpo del operario. El método determina para cada miembro la forma de medición del ángulo, y debe ser aplicado al lado derecho y al lado izquierdo del cuerpo por separado.

Después, las puntuaciones globales de los grupos A y B son modificadas en función del tipo de actividad muscular desarrollada, así como de la fuerza aplicada durante la realización de la tarea. Por último, se obtiene la puntuación final a partir de dichos valores globales modificados.

El valor final dado por el método RULA es proporcional al riesgo que conlleva la realización de la tarea, de forma que valores altos indican un mayor riesgo de aparición de lesiones musculoesqueléticas. El método organiza las puntuaciones finales en niveles de actuación que orientan al evaluador sobre las decisiones a tomar tras el análisis. Los niveles de actuación propuestos van del nivel 1, que estima que la postura evaluada resulta aceptable, al nivel 4, que indica la necesidad urgente de cambios en la actividad.

El diligenciamiento de la entrevista se realizó de manera presencial previa firma del consentimiento informado tanto del operador como del paciente, en un horario que no interfiriera con la actividad que realizaban; la duración aproximada por entrevista fue de 25 minutos.

Para la descripción y el análisis del puesto de trabajo, se utilizó la filmación y la observación directa a partir de cinco visitas al puesto, de aproximadamente una hora cada una.

Para la aplicación del método, se realizó un registro fílmico. Se utilizaron, de manera simultánea, dos videograbadoras Sony en formato MPEG-4 ISO/IEC (Moving Picture Experts Group ${ }^{\circledR}$ ) de alta calidad, con las que se obtuvieron dos videos digitales (el primero desde el plano vertical derecho y el segundo desde el plano vertical izquierdo), los cuales fueron convertidos en imágenes (JPEG) para ser observadas y analizadas en un computador Acer Aspire One 725-0666 mediante el software de licencia libre Kinovea versión 0.8.15, un programa lanzado bajo la licencia GPL en el que se puede cronometrar el tiempo de cada movimiento registrado, explorar la escena cuadro por cuadro, definir líneas de movimiento, ángulos y giros, y calcular el centro de gravedad en determinado momento, entre otros [14], como se puede apreciar en el ejemplo de la figura 1. Es importante mencionar que se filmó una vez a cada sujeto muestra y de manera individual. 


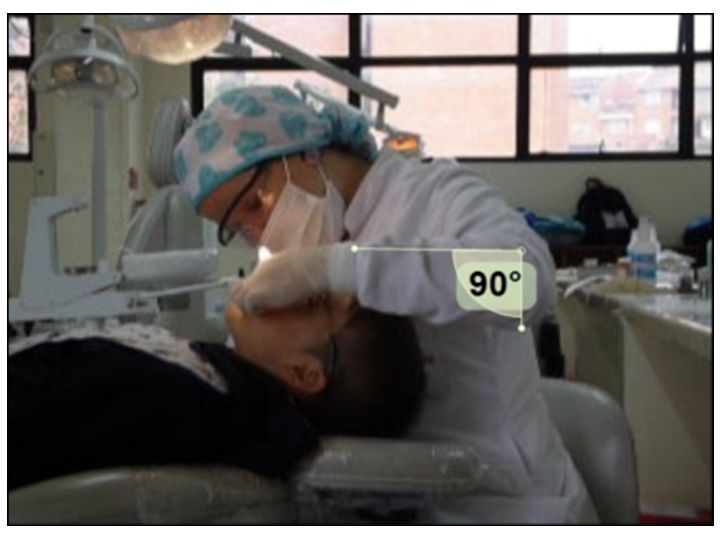

Figura 1. Aplicación del software Kinovea para antebrazo Fuente: elaboración propia

Para hacer el estudio, toda la población muestra se ubicó en la misma unidad odontológica, en el tercer piso de la Escuela de Odontología de la Universidad del Valle, con todos los implementos necesarios: equipos (unidad odontológica), herramientas (micromotor y contraángulo) y materiales de uso odontológico (cepillo de profilaxis y pasta profiláctica). Por último, es relevante indicar que antes de iniciar se calibraron los equipos: altura de los trípodes, distancia focal, campo de observación, resolución de la imagen, resolución del video, zoom óptico y zoom digital; y también se estandarizaron los procedimientos de observación de los videos respecto al método RULA utilizado en este estudio.

\section{Consideraciones éticas}

El diseño de este estudio cumplió con lo estipulado en la Declaración de Helsinki para la investigación en humanos [15] y en la Resolución 008430 del Ministerio de Salud y Protección Social de Colombia [16]; a su vez, obtuvo el aval del Comité Institucional de Revisión de Ética Humana de la Facultad de Salud de la Universidad del Valle.

\section{Análisis estadístico}

Los datos obtenidos fueron ingresados en una plantilla en Excel $^{\oplus}$ y se procesaron en el software SPss $^{\circ}$ versión 17 , mediante análisis univariado y bivariado a través de pruebas de análisis de frecuencias. Es importante mencionar que ambos software cuentan con licencia por parte de la universidad.

\section{Resultados}

Resultados relacionados con el sujeto [17]

La totalidad de la muestra estuvo compuesta por mujeres, el 73\% tenía entre 18 y 23 años de edad, el $100 \%$ reportó haber sufrido traumatismos, sobre todo a nivel de columna vertebral como fisuras, y el 20\% refirió intervención quirúrgica. El 67\%, además de sus actividades académicas, realizó actividades de tipo laboral con jornadas de ocho horas de lunes a viernes y de cuatro horas los sábados. De las personas que trabajan, el $60 \%$ se desempeña como auxiliar de salud oral y el $40 \%$ restante, en ocupaciones relacionadas con trabajo de oficina y ventas.

El $76 \%$ de los sujetos indica molestias físicas, principalmente al finalizar la jornada diaria, en zonas específicas del cuerpo como espalda baja, cabeza, manos y rodillas.

Todos refieren que el tiempo utilizado en la realización del procedimiento de profilaxis oscila entre diez y quince minutos, y reportan como tiempo de descanso cuando el paciente no asiste a la cita del día y los momentos entre un procedimiento y otro o entre un paciente y otro, que constan aproximadamente de dos minutos mientras revisan y diligencian la historia clínica.

Entre los factores que más del $60 \%$ de la muestra considera que influyen en la ejecución del procedimiento de profilaxis están: el material de los equipos, las posiciones corporales que adoptan, la ubicación espacial de las herramientas y el mantenimiento de estas.

Finalmente, el 100\% considera que los factores ambientales que más afectan los procedimientos de salud oral son: las bajas temperaturas y el ruido.

\section{Resultados relacionados con la actividad}

\section{Análisis de la actividad [12]}

Como se observa en la tabla 1, el procedimiento de profilaxis dental se clasifica como actividad de higiene oral 3225, de acuerdo con la clasificación internacional de ocupaciones (CIUO) [18], y consta de tres tareas, once operaciones y catorce pasos.

Se reconoce que la tarea n. $^{\circ} 2$ ("Realizar el procedimiento") es la de mayor duración durante la jornada, con un promedio del 56\%; de igual modo, se considera la más compleja de realizar al tener 
Tabla 1. Descomposición detallada de tareas del procedimiento de profilaxis dental

\begin{tabular}{|c|c|c|c|c|}
\hline Tareas & Operaciones & Pasos & $\begin{array}{c}\text { Porcentaje } \\
\text { de la tarea } \\
\text { en la jornada }\end{array}$ & $\begin{array}{c}\text { Demandas } \\
\text { biomecánicas generales }\end{array}$ \\
\hline $\begin{array}{l}\text { 1. Preparar } \\
\text { puesto de trabajo }\end{array}$ & $\begin{array}{l}\text { 1.1 Alistar materiales de } \\
\text { uso personal } \\
\text { 1.2 Pedir insumos } \\
\text { 1.3 Esterilizar la unidad } \\
\text { odontológica } \\
\text { 1.4 Recibir al paciente }\end{array}$ & $\begin{array}{l}\text { 1.1.1 Ponerse ropa y artefactos de uso } \\
\text { preventivo } \\
\text { 1.2.1 Acercarse a la zona donde les } \\
\text { proporcionan los insumos } \\
\text { 1.3.1 Uso de papel crista flex para cu- } \\
\text { brir zonas y elementos de la unidad } \\
\text { 1.4.1 Llamar al paciente para su ingreso } \\
\text { a la clínica }\end{array}$ & $32 \%$ & $\begin{array}{l}\text { Posición bípeda y ca- } \\
\text { minando. Inclinaciones } \\
\text { laterales de tronco, flexión } \\
\text { de brazos a } 75^{\circ} \text {, flexión de } \\
\text { cadera a } 85^{\circ} \text {, flexión de } \\
\text { codo a } 50^{\circ} \text {, traslaciones } \\
\text { lineales. }\end{array}$ \\
\hline $\begin{array}{l}\text { 2. Realizar el } \\
\text { procedimiento }\end{array}$ & $\begin{array}{l}\text { 2.1 Preparar al paciente } \\
\text { 2.2 Ubicar la manguera } \\
\text { extractora } \\
\text { 2.3 Seleccionar el } \\
\text { dispositivo } \\
\text { 2.4 Seleccionar cuadrante } \\
\text { en la cavidad oral } \\
\text { 2.5 Remover placa } \\
\text { bacteriana }\end{array}$ & $\begin{array}{l}\text { 2.1.1 Ubicar al paciente en la unidad y } \\
\text { sobre él un babero } \\
\text { 2.1.2 Graduar la unidad odontológica } \\
\text { de acuerdo con el usuario } \\
\text { 2.2.1 Introducir extractor en cavidad } \\
\text { oral del paciente } \\
\text { 2.3.1 Uso del cepillo profiláctico } \\
\text { 2.4.1 Iniciar por el cuadrante superior } \\
\text { derecho, luego el superior izquierdo, el } \\
\text { inferior izquierdo y el inferior derecho, } \\
\text { según la dirección de las manecillas del } \\
\text { reloj (recomendación de la Federación } \\
\text { Dental Internacional - FDI) } \\
\text { 2.5.1 Pasar el dispositivo por cada } \\
\text { diente } \\
\text { 2.5.2 Uso de espejo } \\
\text { 2.5.3 Pasar hilo dental por los espacios } \\
\text { entre los dientes }\end{array}$ & $56 \%$ & $\begin{array}{l}\text { Posición bípeda, incli- } \\
\text { nación lateral de tronco, } \\
\text { flexión de brazos } 70^{\circ} \text {. } \\
\text { Posición sedente, flexión } \\
\text { de cuello } 47^{\circ} \text {, inclinación } \\
\text { lateral de tronco, abduc- } \\
\text { ción hombro } 75^{\circ} \text {, flexión } \\
\text { de codo }>100^{\circ} \text {, flexión o } \\
\text { extensión de muñeca }>15^{\circ}\end{array}$ \\
\hline $\begin{array}{l}\text { 3. Finalizar el } \\
\text { procedimiento }\end{array}$ & $\begin{array}{l}\text { 3.1 Retirar la manguera } \\
\text { extractora } \\
\text { 3.2 Graduar el nivel de } \\
\text { la unidad odontológica }\end{array}$ & $\begin{array}{l}\text { 3.1.1 Retirar la manguera de la cavidad } \\
\text { oral y ubicarla en la escupidera } \\
\text { 3.2.1 Descender la unidad, de modo } \\
\text { que el paciente pueda bajar de ella }\end{array}$ & $12 \%$ & $\begin{array}{l}\text { Posición sedente, flexión } \\
\text { de brazo } 60^{\circ} \text {, flexión } \\
\text { de muñeca } 54^{\circ} \text {, agarre } \\
\text { penta digital }\end{array}$ \\
\hline
\end{tabular}

Fuente: Elaboración propia

el mayor número de operaciones y pasos (cinco y ocho, respectivamente), y también es la de mayor demanda biomecánica, pues exige al operador flexión de cuello, inclinación lateral de tronco, abducción del hombro, flexión del codo y flexión y/o extensión de la muñeca. Debido a esto, es la tarea crítica, es decir, la de mayor potencial de producir daño por su complejidad, el tiempo de exposición y la demanda física.

\section{Secuencia}

El $80 \%$ realiza los mismos pasos, pero en orden diferente a los que se establecen en el análisis de la actividad (tabla 1, paso 2.4.1). Mientras en el análisis se plantea que el trabajo debe ser en el sentido de las manecillas del reloj, iniciando por el cuadrante superior derecho, los estudiantes que participaron en el estudio inician por los cuadrantes superiores y después pasan a los inferiores, lo que puede reflejar un ajuste para facilitar su trabajo al abordar primero lo más externo en la cavidad oral del paciente. El 20\% restante omite pasos o adiciona otros al procedimiento.

\section{Ritmo de tiempo}

El tiempo se tomó solo para la tarea crítica, es decir, la n. ${ }^{\circ} 2$; la ejecución de la tarea fue variable entre un sujeto y otro, oscilando entre veinte y veintiocho minutos, con promedio efectivo de veinticuatro minutos. Entre los factores que interfirieron con el ritmo están: aquellos propios de la tarea, los medioambientales y los personales.

\section{Acciones requeridas y destrezas de ejecución}

El procedimiento exige al operador permanecer aproximadamente el $68 \%$ del tiempo en posición sedente, con una carga postural estática a nivel de 
hombros y codos, y con movimiento sobre todo en la articulación de la muñeca y el cuello. La tarea n. ${ }^{\circ}$ 2 exige estar todo el tiempo en posición sedente, realizando un trabajo minucioso de precisión que se ejecuta con ayuda de pequeños movimientos de escasa amplitud, en un campo muy pequeño, donde además está la lengua, la saliva y hay que introducir una serie de instrumentos.

Otras destrezas que se demandan son: cierre visual, agudeza visual, enfoque, atención al detalle, procesamiento sensorio-motor conservado, procesamiento cognitivo y de memoria, atención, secuenciación, orientación y nivel de conciencia. A nivel psicosocial, se requiere asertividad en solución de conflictos, autocontrol, ejecución de su rol y organización del tiempo.

\section{Resultados relacionados con el medio ambiente $[19,20]$}

La clínica donde se trabajó cuenta con veinticuatro unidades dentales, lavamanos horizontales y otros mobiliarios que hacen que el espacio sea reducido, y dificultan la circulación libre entre las unidades odontológicas y las zonas de paso. La distancia entre la unidad odontológica y las divisiones (lavamanos horizontales) es de $1 \mathrm{~m}$, pero respecto a la silla es de $26 \mathrm{~cm}$, lo que hace que no se pueda acceder a los lavamanos y que se interfiera con las acomodaciones que realiza el operador para acceder a la cavidad oral del paciente.

Las condiciones inadecuadas de la silla del operador (acolchado de la silla deteriorado, espaldar sin graduación y rodachines en mal estado) causan posturas incorrectas y adormecimiento de los miembros inferiores por interrupciones parciales del oxígeno hacia los músculos y por presión sobre la zona poplítea, lugar donde ejerce presión el borde la silla [21].

La iluminación se identifica como deficiente, situación que puede deberse a una variedad de factores, entre ellos: reducción de la intensidad de salida de las unidades de luz por una caída del voltaje de la línea, degradación o desgaste del bombillo, reflectores, filtros perforados o fracturados y variaciones en el diseño [22], o a la mala ubicación de la lámpara, relativamente lejos de la línea de visión del profesional al momento de ejecutar el procedimiento. Para esta investigación, se considera que la deficiencia se debe a la reducción de la intensidad de luz por degradación o desgaste del bombillo.

El espacio maneja bajas temperaturas, pues el aire acondicionado está graduado entre 16 y $22^{\circ} \mathrm{C}$, y los ductos de distribución caen directamente sobre las unidades odontológicas, generando incomodidad para los pacientes y los operadores. Por otra parte, el ruido de los dispositivos como piezas de baja velocidad, accionados de forma simultánea por diferentes operadores en la clínica, genera interferencias en la comunicación con los pacientes, los compañeros, y el personal docente y administrativo.

Finalmente, se evidencia que es frecuente que ocurran daños inesperados en los equipos, dispositivos y herramientas de trabajo.

\section{Resultados aplicación del método RULA}

Como se observa en la tabla 2, la puntuación predominante para brazo fue de 3 tanto para el hemicuerpo izquierdo como para el derecho, con $93 \%$ y $83 \%$, respectivamente. Esto pone de manifiesto que se realiza flexión en el rango de $20-45^{\circ}$ y abducción del brazo sin punto de apoyo, lo que no permite que se reduzca la carga postural y se refleja en un incremento del riesgo por la posición de este segmento contra la gravedad. Es importante resaltar que este segmento solo cuenta con apoyo a nivel distal, específicamente en la cavidad oral del paciente (dientes de los cuadrantes superiores o inferiores lado izquierdo). La ligera diferencia de los hemicuerpos radica en que existen sujetos que con el hemisferio derecho realizan flexiones menores de $20^{\circ}$ y no hacen abducción.

Para el antebrazo, se observa en la tabla 2 que el 100\% de la muestra obtiene una puntuación de 3 en los dos hemisferios, lo que significa que se realiza el movimiento de flexión por encima de los $100^{\circ}$, con proyección vertical del antebrazo más allá del codo. Estas cifras son significativas, porque indican carga postural o posición mantenida de este segmento corporal durante la mayor parte del tiempo mientras se realiza la profilaxis.

El 100\% de los operadores tuvo una puntuación de 4 para los dos hemisferios en el segmento de muñeca, es decir que realizan extensión o flexión por encima de los $15^{\circ}$, y además se modifica la calificación, incrementando un punto por realizar desviación ulnar o radial en el procedimiento y un punto más por realizar giro de muñeca pronación o supinación en el rango medio. Esto se 
Tabla 2. Puntuación global grupo $\mathrm{A}^{\star}$

\begin{tabular}{|c|c|c|c|c|c|c|c|c|c|c|}
\hline \multirow[t]{2}{*}{$\begin{array}{l}\text { Sujetos } \\
\text { muestra }\end{array}$} & \multicolumn{2}{|c|}{ Puntuación brazo } & \multicolumn{2}{|c|}{$\begin{array}{l}\text { Puntuación } \\
\text { antebrazo }\end{array}$} & \multicolumn{2}{|c|}{$\begin{array}{l}\text { Puntuación } \\
\text { muñeca }\end{array}$} & \multicolumn{2}{|c|}{$\begin{array}{c}\text { Puntuación global } \\
\text { grupo A }\end{array}$} & \multicolumn{2}{|c|}{$\begin{array}{l}\text { Puntuación global } \\
\text { grupo A } \\
\text { + modificación }\end{array}$} \\
\hline & HI & HD & HI & HD & HI & HD & HI & HD & HI & HD \\
\hline 1 & 3 & 3 & 3 & 3 & 5 & 5 & 5 & 5 & $5+1=6$ & $5+1=6$ \\
\hline 2 & 3 & 1 & 3 & 3 & 5 & 4 & 5 & 4 & $5+1=6$ & $4+1=5$ \\
\hline 3 & 2 & 1 & 3 & 3 & 5 & 4 & 5 & 4 & $5+1=6$ & $4+1=5$ \\
\hline 4 & 3 & 4 & 3 & 3 & 5 & 6 & 5 & 6 & $5+1=6$ & $6+1=7$ \\
\hline 5 & 3 & 3 & 3 & 3 & 5 & 5 & 5 & 5 & $5+1=6$ & $5+1=6$ \\
\hline 6 & 3 & 3 & 3 & 3 & 5 & 5 & 5 & 5 & $5+1=6$ & $5+1=6$ \\
\hline 7 & 3 & 3 & 3 & 3 & 5 & 5 & 5 & 5 & $5+1=6$ & $5+1=6$ \\
\hline 8 & 3 & 3 & 3 & 3 & 5 & 5 & 5 & 5 & $5+1=6$ & $5+1=6$ \\
\hline 9 & 3 & 3 & 3 & 3 & 5 & 5 & 5 & 5 & $5+1=6$ & $5+1=6$ \\
\hline 10 & 3 & 3 & 3 & 3 & 5 & 5 & 5 & 5 & $5+1=6$ & $5+1=6$ \\
\hline 11 & 3 & 3 & 3 & 3 & 5 & 5 & 5 & 5 & $5+1=6$ & $5+1=6$ \\
\hline 12 & 3 & 3 & 3 & 3 & 5 & 5 & 5 & 5 & $5+1=6$ & $5+1=6$ \\
\hline 13 & 3 & 2 & 3 & 3 & 5 & 4 & 5 & 4 & $5+1=6$ & $4+1=5$ \\
\hline 14 & 3 & 2 & 3 & 3 & 5 & 4 & 5 & 4 & $5+1=6$ & $4+1=5$ \\
\hline 15 & 3 & 3 & 3 & 3 & 5 & 5 & 5 & 5 & $5+1=6$ & $5+1=6$ \\
\hline Frecuencia & $\begin{array}{c}14=3 \\
1=2\end{array}$ & $\begin{array}{l}1=4 \\
9=3 \\
2=2 \\
2=1\end{array}$ & $15=3$ & $15=3$ & $15=5$ & $\begin{array}{c}1=6 \\
10=5 \\
4=4\end{array}$ & $15=5$ & $\begin{array}{c}1=6 \\
10=5 \\
4=4\end{array}$ & $15=6$ & $\begin{array}{c}1=7 \\
10=6 \\
4=5\end{array}$ \\
\hline Porcentaje & $\begin{array}{c}\mathbf{3}=\mathbf{9 3} \% \\
2=7 \%\end{array}$ & $\begin{array}{c}4=7 \% \\
2=10 \% \\
3=\mathbf{3 3} \%\end{array}$ & $3=100 \%$ & $3=100 \%$ & $5=100 \%$ & $\begin{array}{c}4=27 \% \\
6=7 \%\end{array}$ & $5=100 \%$ & $\begin{array}{c}4=27 \% \\
6=7 \% \\
5=66 \%\end{array}$ & $6=100 \%$ & $\begin{array}{l}5=27 \% \\
7=7 \% \\
\mathbf{6}=\mathbf{6 6} \%\end{array}$ \\
\hline
\end{tabular}

${ }^{\star}$ Grupo A: hemicuerpo izquierdo (HI); hemicuerpo derecho (HD).

Fuente: elaboración propia

traduce en una de las mayores cargas posturales de los miembros superiores. Es relevante mencionar que la dominancia derecha de los participantes y la mayor demanda que tiene la actividad para el segmento derecho pueden explicar que a pesar de que la calificación está en los mismos rangos de la muñeca izquierda, los movimientos de la muñeca derecha son más pronunciados y aumentan aún más la carga postural identificada.

Finalmente, se obtiene una puntuación de 5 para el $100 \%$ del grupo de segmentos del hemicuerpo izquierdo y para el $66 \%$ del hemicuerpo derecho; se modifica la calificación añadiendo un un punto, debido a que la postura que asume el segmento es principalmente estática, con agarres superiores a un minuto y realizando la acción repetidamente (cuatro veces por minuto o más). No se añade puntuación por fuerza o carga, ya que esta es menor a $2 \mathrm{~kg}$ e intermitente; por lo tanto, la puntuación final del grupo A para los miembros superiores es de 6 .

En la tabla 3, se observa que la puntuación para cuello fue de 4 en el $100 \%$ de la población. Esto se debe a que los operadores hacen una flexión del cuello mayor a $20^{\circ}$ y también realizan una inclinación lateral del cuello, poniendo este segmento corporal en posición mantenida durante un tiempo prolongado.

Así mismo, la tabla muestra para tronco una puntuación de 4 en el 53\% de los operadores, es decir que realizan flexión en el rango de 20 y $60^{\circ}$ e inclinación lateral, exponiendo este segmento a posición sostenida durante la ejecución de la profilaxis.

La posición y la consecuente puntuación de cuello y tronco se pueden explicar como una forma que tienen los operadores de realizar ajustes 
Tabla 3. Puntuación global del grupo B

\begin{tabular}{|c|c|c|c|c|}
\hline Sujetos muestra & Puntuación cuello & Puntuación tronco & Puntuación piernas & $\begin{array}{c}\text { Puntuación final } \\
\text { grupo B }\end{array}$ \\
\hline 1 & 4 & 4 & 2 & 7 \\
\hline 2 & 4 & 3 & 2 & 7 \\
\hline 3 & 4 & 2 & 2 & 6 \\
\hline 4 & 4 & 3 & 2 & 7 \\
\hline 5 & 4 & 4 & 2 & 7 \\
\hline 6 & 4 & 4 & 2 & 7 \\
\hline 7 & 4 & 4 & 2 & 7 \\
\hline 8 & 4 & 3 & 2 & 7 \\
\hline 9 & 4 & 3 & 2 & 7 \\
\hline 10 & 4 & 4 & 2 & 7 \\
\hline 11 & 4 & 4 & 2 & 7 \\
\hline 12 & 4 & 4 & 2 & 7 \\
\hline 13 & 4 & 3 & 2 & 7 \\
\hline 14 & 4 & 4 & 2 & 7 \\
\hline 15 & 4 & 3 & 2 & 7 \\
\hline Frecuencia & $15=4$ & $8=4 / 6=3 / 1=2$ & $15=2$ & $14=7 / 1=6$ \\
\hline Porcentaje & $4=100 \%$ & $\mathbf{4}=\mathbf{5 3} \% / 3=40 \% / 2=7 \%$ & $2=100 \%$ & $7=93 \% / 6=7 \%$ \\
\hline
\end{tabular}

${ }^{*}$ Grupo B: cuello, tronco y piernas

Fuente: elaboración propia

posturales para acceder mejor a la cavidad oral del paciente y responder a las altas demandas visuales del procedimiento.

En relación con las piernas, estas puntuaron en 2 para el $100 \%$ de los operadores, debido a que las piernas y los pies no están apoyados ni equilibrados a pesar de encontrarse en posición sedente.
Finalmente, la puntuación global para el grupo B fue de 7 para el $93 \%$.

En la tabla 4, se observa una puntuación global final de 7 , que se obtiene de cruzar la puntuación obtenida en el grupo A de 6 y la obtenida en el grupo B de 7 .

Tabla 4. Puntuación global final

\begin{tabular}{|c|c|c|c|c|c|c|c|}
\hline \multirow[t]{2}{*}{$\begin{array}{l}\text { Puntuación } \\
\text { (grupo A) }\end{array}$} & \multicolumn{7}{|c|}{ Puntuación (grupo B) } \\
\hline & 1 & 2 & 3 & 4 & 5 & 6 & 7 \\
\hline 1 & 1 & 2 & 3 & 3 & 4 & 5 & 5 \\
\hline 2 & 2 & 2 & 3 & 4 & 4 & 5 & 5 \\
\hline 3 & 3 & 3 & 3 & 4 & 4 & 5 & 6 \\
\hline 4 & 3 & 3 & 3 & 4 & 5 & 6 & 6 \\
\hline 5 & 4 & 4 & 4 & 5 & 6 & 7 & 7 \\
\hline 6 & 4 & 4 & 5 & 6 & 6 & 7 & 7 \\
\hline 7 & 5 & 5 & 6 & 6 & 7 & 7 & 7 \\
\hline 8 & 5 & 5 & 6 & 7 & 7 & 7 & 7 \\
\hline
\end{tabular}

Fuente: Método de evaluación RULA 
Tabla 5. Niveles de actuación según puntuación final obtenida

\begin{tabular}{cl}
\hline Nivel & \multicolumn{1}{c}{ Actuación } \\
\hline $\mathbf{1}$ & $\begin{array}{l}\text { Cuando la puntuación final es } 1 \text { o 2: la postura es } \\
\text { aceptable }\end{array}$ \\
$\mathbf{2}$ & $\begin{array}{l}\text { Cuando la puntuación final es } 3 \text { o } 4 \text {, pueden reque- } \\
\text { rirse cambios en la tarea: es conveniente profundizar } \\
\text { en el estudio }\end{array}$ \\
& $\begin{array}{l}\text { La puntuación final es } 5 \text { o } 6: \text { se requiere el rediseño } \\
\text { de la tarea; es necesario realizar actividades de } \\
\text { investigación }\end{array}$ \\
& $\begin{array}{l}\text { La puntuación final es } 7: \text { se requieren cambios urgen- } \\
\text { tes en el puesto o tarea }\end{array}$ \\
\hline
\end{tabular}

Fuente: Método de evaluación RULA

En la tabla 5, se observa que después de ingresar la puntuación global final de 7, el nivel de actuación está ubicado en 4; es decir que el puesto de trabajo y la tarea crítica requieren cambios urgentes, debido a la exposición a factores de riesgo que pueden producir trastornos en la salud, especialmente en el sistema musculoesquelético de la población analizada.

\section{Discusión}

La investigación reveló que los estudiantes del programa de Auxiliar de Salud Oral están expuestos, en mayor medida, a trastornos del sistema musculoesquelético como consecuencia de la relación dinámica entre actividad, lugar de trabajo y adopción de posturas inadecuadas.

Estos trastornos se convierten en el peligro común de esta población, con efectos nocivos para la salud que van desde malestar, dolor persistente en las articulaciones, músculos y tendones, hasta la enfermedad profesional y posible situación de discapacidad. En este sentido, el resultado del estudio arroja la necesidad de efectuar cambios urgentes en el puesto de trabajo, y confirma estudios realizados por Alexopoulos et al. [10] y Dayakar et al. [23], entre otros.

Aunque las causas de un trastorno musculoesquelético no se pueden atribuir a un único factor de riesgo, este estudio reconoce los factores de riesgo más relevantes que están presentes en la relación dinámica entre actividad, medio ambiente y sujeto que ejecuta el trabajo.
El factor que más se destaca aquí es el asociado a la adopción de posturas inadecuadas de los operadores, caracterizada por: i) flexión de brazo en el rango de $20-45^{\circ}$ y abducción del brazo con punto de apoyo solo a nivel distal, específicamente en la cavidad oral del paciente; ii) antebrazo con movimiento de flexión por encima de $100^{\circ} \mathrm{y}$ con proyección vertical del antebrazo más allá de codo, sin apoyo; iii) muñeca con movimiento de flexión o extensión mayor de $15^{\circ}$ y desviación ulnar o radial; iv) cuello con flexión mayor de $20^{\circ}$ e inclinación lateral; v) tronco con flexión en el rango de $20-60^{\circ}$ e inclinación lateral; y vi) piernas sin apoyo y equilibrio.

Estos resultados se confirman con el estudio realizado por Morán [24] sobre posturas ergonómicas adoptadas por profesionales de odontología, en el que se evidencia que la no adopción de posturas ergonómicas genera síntomas osteomusculares en columna vertebral y en miembros superiores; y con el estudio de Mendoza [25], en el que evalúa las posturas inadecuadas adoptadas por odontólogos durante sus actividades laborales y las relaciona con molestias principalmente en cuello y zona lumbar, y además estableció un nivel de riesgo alto para la población estudiada y concluyó que la existencia de desórdenes por trauma acumulativo en los odontólogos es resultado de las posturas inadecuadas que se adoptan durante los procedimientos propios de la actividad laboral, ya que la correcta postura le resulta un tanto incómoda a dicho personal [25].

La postura identificada en esta investigación es adoptada por los auxiliares para responder a las demandas de la actividad como: trabajar con las manos cerca de la cara, lograr una buena visión de la boca, capacidad de acceso a la cavidad oral, alta precisión, movimientos pequeños de escasa amplitud, mantener la empuñadura de los instrumentos, falta de soportes en los antebrazos, luz de la unidad odontológica reducida, espacio estrecho de movilidad y baja temperatura. Este resultado se contrasta con el de Chávez et al. [26], quienes observaron una alta ocurrencia de trastornos musculoesqueléticos en el desempeño de sus actividades como odontólogos, por tener que realizar trabajo repetitivo, posturas inadecuadas, vibración, etc., y concluyeron que cada músculo, nervio o tendón que intervino en el trabajo clínico se vio afectado por los riesgos ergonómicos. Así, la prevención dependerá de la capacitación y del conocimiento sobre posturas odontológicas ergonómicas [26]. 
Otro resultado del estudio indica que las posturas de los diferentes segmentos fueron predominantemente estáticas durante el desarrollo del procedimiento de profilaxis (sentado durante el $62 \%$ del tiempo); esto añade otra variable que se suma a la postura inadecuada que se encontró como predominante para el grupo objeto de estudio. Estas posturas mantenidas de manera prolongada generan fatiga que se va acumulando en los diferentes segmentos, y un deterioro progresivo y alteraciones fisiopatológicas del sistema musculoesquelético, que inician como molestias de aparición lenta y de carácter inofensivo, pero que se hacen crónicas desarrollando un daño permanente.

\section{Conclusiones}

La investigación concluye que los estudiantes del programa de Auxiliar de Salud Oral tienen un factor de riesgo importante de desarrollar desórdenes musculoesqueléticos en cuello, en parte baja de la espalda y en miembros superiores, especialmente en el segmento de muñeca; y que es necesario un cambio urgente en su puesto de trabajo, pues de continuar realizando la actividad de profilaxis dental como hasta el momento, con el tiempo sobrevendrá inevitablemente una enfermedad en el sistema musculoesquelético.

La postura sentada es la posición de trabajo más confortable, ya que ayuda a reducir la fatiga corporal, disminuye el gasto de energía e incrementa la estabilidad y la precisión en las acciones desarrolladas. Sin embargo, esta postura también puede resultar perjudicial para la salud si no se tienen en cuenta los principales requisitos ergonómicos de los elementos que intervienen en la realización del trabajo, especialmente la silla, la unidad odontológica y el plano de trabajo. También resulta perjudicial si se mantiene durante mucho tiempo, por lo que es aconsejable alternarla con la postura de pie y caminando.

Es necesario un programa preventivo que aborde la capacitación y el entrenamiento en posturas ergonómicas en el desempeño de cada una de las tareas que realiza el estudiante auxiliar de salud oral, pero especialmente en la tarea crítica de realizar el procedimiento de profilaxis.

\section{Recomendaciones}

- Implementar estrategias de promoción y prevención que ayuden a minimizar el impacto de los factores de riesgo en la salud de los auxiliares de salud oral.

- Abordar la unidad odontológica en posición sedente, con el fin de minimizar el impacto de los aspectos biomecánicos, de tal forma que el operador logre una postura equilibrada, cómoda y apoyada, tal como lo indica la Organización Mundial de la Salud (oms), con base en lo que Beach [27] ha denominado Balanced Home Operating Position o posición de máximo equilibrio o posición cero, ya que permite al odontólogo (en este caso, auxiliares de salud oral) realizar su trabajo con el mayor número de músculos en semirrelajación:

1. La cabeza ligeramente inclinada, con el fin de ver bien el campo operatorio del interior de la boca del paciente (la boca del paciente debe coincidir con el plano sagital o medio del operador, cualquier desviación de dicho plano obliga a realizar giros o torsiones del cuello o de la espalda).

2. Los hombros paralelos al plano horizontal y la espalda recta (se establece un perfecto equilibrio del cuerpo, queda completamente balanceado entre sus dos mitades y al tener la espalda recta, se ayuda a mantener la lordosis fisiológica lumbar y a disminuir la presión sobre los discos intervertebrales y la sobrecarga postural de los músculos de la espalda).

3. Los brazos pegados al cuerpo (con el fin de no fatigar los músculos de cuello y hombros y tener un buen apoyo; además los codos deben estar bajos, pegados a la parte costal).

4. Las manos a la altura de la línea media sagital del esternón, por ser este el punto de trabajo (a este nivel y a la altura de la punta del esternón es donde debe estar la boca del paciente). Para que esto se cumpla, se recomienda que la cabeza del paciente esté en un punto intermedio entre el corazón y el ombligo.

5. Los muslos paralelos al plano del suelo (el ángulo formado por la columna vertebral y el fémur debe ser superior a los $100^{\circ}$, ya que por debajo de esta cifra se tiende a rectificar la lordosis lumbar; se admite que los muslos 
estén paralelos al plano del suelo y que formen con la espalda un ángulo de $90^{\circ}$ ).

6. Los pies apoyados en el suelo y ligeramente separados, ya que soportan un $25 \%$ de la carga del peso del cuerpo.

- Implementar descansos durante la jornada laboral. Las directrices de la Applied Occupational and Environmental Hygiene [28] recomiendan descansos de, por lo menos, seis minutos cada hora a los trabajadores que realizan movimientos repetitivos, de tal forma que se debe diseñar un plan de trabajo diario que incluya procedimientos complementarios y variados para así permitir el descanso de grupos musculares alternos y continuar manteniendo un trabajo productivo.

- Desarrollar la función de estiramiento de los músculos y estructuras tendinosas en la preparación del cuerpo para la actividad, así como promover el papel del ejercicio físico como elemento clave y efectivo en el fortalecimiento del torso.

- Seguir los lineamientos de la Iso 6385 [29], que constituyen el estándar sobre los principios ergonómicos para el diseño de sistemas de trabajo, aplicables al diseño del equipo odontológico, al tener en cuenta el área de trabajo, el diseño en relación con la postura, la fuerza muscular, los movimientos del cuerpo humano y el diseño con el ambiente.

- Realizar el mantenimiento preventivo de los equipos e instrumentos, así como trabajar con una silla ergonómica, un sillón dental graduable, una iluminación adecuada y el uso de sistemas tales como lupas y microscopios especializados que ayuden a implementar una buena postura y a reducir la fatiga.

\section{Referencias}

[1] Fabiani I. Prevalencia de patología músculo-esquelética reumatoidea en el Cesfam. Santiago de Chile: Universidad de Chile; 2006. Disponible en: http:// www.repositorio.uchile.cl/handle/2250/110645

[2] Kumar R, Kumar S. Musculoskeletal risk factors in cleaning occupation - A literature review. Int J Ind Ergonom. 2008;38(2):158-70. doi: http://doi.org/ 10.1016/j.ergon.2006.04.004

[3] Kumar S. Perspectives in rehabilitation ergonomics. United Kingdom: CRC Press; 2003.
[4] Colombia, Ministerio de Salud y Protección Social. Informe de enfermedad profesional en Colombia 2001-2002: una oportunidad para la prevención. Bogotá: Ministerio de Salud y Protección Social; 2004.

[5] Cruz A, Garnica A. Ergonomía aplicada: Bogotá: Ecoe; 2011.

[6] Sánchez M. Prevención de riesgos del personal de salud. Medwave. 2004;4(6):e2790. doi: 10.5867/medwave.2004.06.2790

[7] Apud E, Meyer F. La importancia de la ergonomía para los profesionales de la salud. Ciencia y Enfermería. 2003;9(1):15-20. doi: http://dx.doi.org/10.40 67/S0717-95532003000100003

[8] Otero JJG. Riesgos del trabajo del personal sanitario. Madrid: McGraw-Hill; 1993.

[9] Bugarín-González R, Galego-Feal P, García-García A, Rivas-Lombardero P. Los trastornos musculoesqueléticos en los odontoestomatólogos. RCOE. 2005; 10(5-6):561-6.

[10] Alexopoulos EC, Stathi I-C, Charizani F. Prevalence of musculoskeletal disorders in dentists. BMC Musculoskeletal Disorders. 2004;5(1):16. doi: 10.11 86/1471-2474-5-16

[11] Bendezú Aguirre NV, Valencia Tapia E, Aguilar Mendoza LA, Fonseca V, Cecilia N. Correlación entre nivel de conocimientos sobre posturas odontológicas ergonómicas, posturas de trabajo y dolor postural según zonas de respuesta, durante las prácticas clínicas de estudiantes en una Facultad de Estomatología. Rev Estomatol Hered. 2006;16(1):26-32.

[12] Fernández-Ríos M. Análisis y descripción de puestos de trabajo: teoría, métodos y ejercicios. Madrid: Ediciones Díaz de Santos; 1995.

[13] McAtamney L, Corlett EN. RULA: A survey method for the investigation of work-related upper limb disorders. Applied Ergonomics. 1993;24(2):91-9. doi: https://doi.org/10.1016/0003-6870(93)90080-S

[14] Sofware libre Kinovea. Kinovea 0.8.15. Un microscopio para sus videos [Internet]. 2007 [citado 2016 julio 2]. Disponible en: www:/kinovea.org.

[15] Manzini JL. Declaración de Helsinki: Principios éticos para la investigación médica sobre seres humanos. Acta Bioeth. 2000;6(2):122-4. doi: http://dx. doi.org/10.4067/S1726-569X2000000200010

[16] Colombia, Ministerio de Salud y Protección Social. Resolución 008430 de 1993, Por la cual se establecen las normas científicas, técnicas y administrativas para la investigación en salud (04 oct 1993).

[17] Asensio-Cuesta S, Ceca MJB, Más JAD. Evaluación ergonómica de puestos de trabajo. Madrid: Paraninfo; 2012. 
[18] OIT - Organización Internacional del Trabajo. Clasificación internacional uniforme de ocupaciones CIUO-88. Ginebra: OIT; 1992.

[19] Pujol J. Análisis ocupacional. Manual de Aplicación para Instituciones de Formación Profesional. Montevideo: Cinterfor/ort; 1987.

[20] Montoya Díaz M, Palucci Marziale MH, Do Carmo Cruz Robazzi ML, Taubert de Freitas FC. Lesiones osteomusculares en trabajadores de un hospital mexicano y la ocurrencia del ausentismo. Ciencia y Enfermería. 2010;16(2):35-46. doi: http://dx.doi. org/10.4067/S0717-95532010000200005

[21] Pedro M, Enrique G, Joan B, Pedro B. Ergonomía 3: diseño de puestos de trabajo. México: Editorial Alfaomega; 2001.

[22] López Soto OP, Acebedo Vallejo JE, Joya Rodríguez LD, López Macías AM. Evaluación de la intensidad de salida de la luz de las lámparas de fotocurado de una clínica dental. Rev Colomb Investig Odontol. 2011;2(4):24-32.

[23] Dayakar M, Gupta S, Philip G, Pai P. Prevalence of musculoskeletal disorder among dental practitioners. ASL-Musculoskeletal. Diseases. 2013;1(1): 22-5.
[24] Morán W, De D’Pool JF. Posturas ergonómicas adoptadas por profesionales de la Odontología. Investigación Social en Salud. 2014;2(2):135-49.

[25] Mendoza-Martinez E. Evaluación de desórdenes de trauma acumulativo músculo esquelético en Odontólogos [tesis doctoral]. [México:] Instituto Politécnico Nacional; 2010.

[26] Chávez López R, Preciado Serrano MDL, Colunga Rodríguez C, Mendoza Roaf PL, Aranda Beltrán C. Trastornos músculo-esqueléticos en odontólogos de una institución pública de Guadalajara, México. Cienc Trab. 2009;11(33):152-5.

[27] Robinson M. Home Position Dentistry (the Beach philosophy of dental practice). Kyoto: Bikenssha Company; 1976.

[28] Mignone Jr. AT, Beckhusen EC, O’Leary K, Gochfeld M, Robbins SK, Blehm KD, et al. Applied occupational and environment hygiene. J Appl Occup Environ Hyg. 1990;5(7):399.

[29] Icontec. Norma Técnica Colombiana NTC-ISO 5655: 2008, Principios para el diseño ergonómico de sistemas de trabajo. Bogotá: Icontec; 2008. 